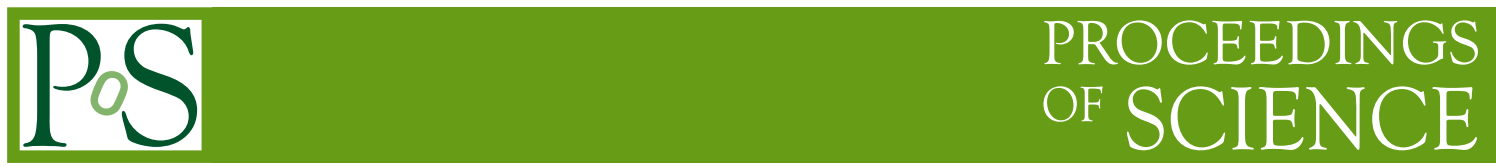

\title{
Charm Decays and Spectroscopy at BABAR
}

\author{
Romulus Godang*† \\ On Behalf of the BABAR Collaboration \\ Department of Physics \\ University of South Alabama \\ ILB 115, 307 University Blvd., $N$. \\ E-mail: godang@usouthal.edu
}

SLAC-PUB-15329

We present searches for rare charm decays of the form $X_{c}^{+} \rightarrow h^{ \pm} \ell^{\mp} \ell^{()^{+}}$, where $X_{c}^{+}$is a charm hadron either $D^{+}, D_{s}^{+}$, or $\Lambda_{c}^{+}$, and $\ell^{()^{ \pm}}$is an electron or muon. These modes are based on 384 $f b^{-1}$ of $e^{+} e^{-}$annihilation data collected at the $\Upsilon(4 S)$ resonance with the BABAR detector at the SLAC National Accelerator Laboratory. We also present the flavor-changing neutral-current decays $D^{0} \rightarrow e^{+} e^{-}, D^{0} \rightarrow \mu^{+} \mu^{-}$, and $D^{0} \rightarrow e^{ \pm} \mu^{\mp}$ that corresponds to an integrated luminosity of $468 \mathrm{fb}^{-1}$ of data. The decay $D^{0} \rightarrow e^{+} \mu^{-}$is further lepton-flavor violating, and thus occur only through very slow neutrino mixing. These decays constitute sensitive probes for possible new-physics contribution. We report new limits on the branching fractions of these decays.

36th International Conference on High Energy Physics,

July 4-11, 2012

Melbourne, Australia

\footnotetext{
* Speaker.

${ }^{\dagger}$ This work was supported by the U.S. Department of Energy under grant No. DE-FG02-96ER-40970
} 


\section{INTRODUCTION}

In the Standard Model (SM), the flavor-changing neutral processes are very rare and are of obvious interest in the search for new physics. In the Flavor-Changing Neutral Current (FCNC) decays $D^{0} \rightarrow \ell^{+} \ell^{-}$, where $\ell$ is an either electron or muon, are strongly suppressed by the GlashowIliopoulos-Maiani (GIM) mechanism [1]. These decays cannot occur at tree level in the SM. The branching fraction of the decays $D^{0} \rightarrow \ell^{+} \ell^{-}$are predicted to be $\mathscr{O}\left(10^{-13}\right)$ [2].

Most of the attention on FCNC decays has been focused in the $K$ and $B$ meson sectors and less in the charm meson sector. It is due to the fact that the SM expectations for $D^{0}-\bar{D}^{0}$ mixing are very small compared to the $K^{0}-\bar{K}^{0}$ and $B^{0}-\bar{B}^{0}$ mixing. However, the FCNC decay in the charm sector is unique due to its decays involve an up-type quark which implies into an effective GIM cancellations and new physics. The decay modes of Lepton-Flavor Violating (LFV) which corresponding to two leptons with two oppositely charged of different flavor and Lepton-Number Violating (LNV) decays where two leptons have the same charge are forbidden in the SM.

Figure 1 shows the Standard Model short-distance contributions to the $c \rightarrow u \ell^{+} \ell^{-}$transition. The branching fraction for the decay of $D \rightarrow X_{u} \ell^{+} \ell^{-}$is predicted to be $\mathscr{O}\left(10^{-8}\right)[2,3]$. The decay of $c \rightarrow u \ell^{+} \ell^{-}$is screened by the long distance contributions. It is also expected to dominate over the short distance contributions in $D^{0}-\bar{D}^{0}$ mixing. The long distance contributions were shown to be largely dominant in $c \rightarrow u \ell^{+} \ell^{-}$. The experimental upper bounds on the branching fraction of $c \rightarrow u \ell^{+} \ell^{-}$is presently in the range of $\mathscr{O}\left(10^{-5}\right)$ [4]. It is an order of magnitude larger than the Standard Model prediction for specific channels [5]. The highest rate of $D \rightarrow V \ell^{+} \ell^{-}$channel with $V=\rho, \omega, \phi, K^{*}$ is the decay of $D_{s}^{+} \rightarrow \rho^{+} \ell^{+} \ell^{-}$. It is predicted at the highest rate $\approx 3 \times 10^{-5}$, however the there are unfortunately no experimental data on this channel.
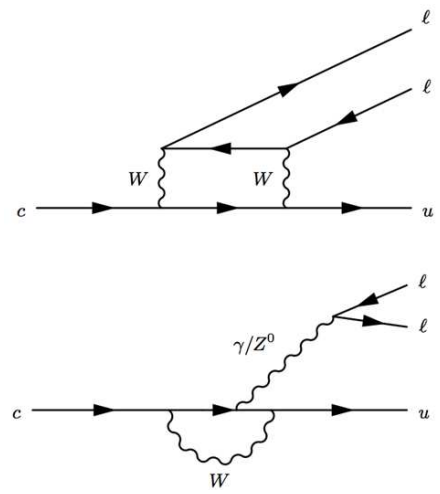

Figure 1: Standard Model short-distance contributions to the $c \rightarrow u \ell^{+} \ell^{-}$transition.

\section{The BABAR DETECTOR AND DATA SET}

The BABAR detector was operated at the PEP-II asymmetric-energy storage rings at the SLAC National Accelerator Laboratory. The data used in this analysis were collected with the BABAR detector. The data sample consist of an integrated luminosity of $384 \mathrm{fb}^{-1}$ for $X_{c}^{+} \rightarrow h^{ \pm} \ell^{\mp} \ell^{\left({ }^{\prime}\right)^{+}}$ and $468 \mathrm{fb}^{-1}$ for $D^{0} \rightarrow \ell^{+} \ell^{-}$accumulated at the $\Upsilon(4 S)$ resonance and $40 \mathrm{MeV}$ below the $\Upsilon(4 S)$ 
resonance. The asymmetric energy of the PEP-II $e^{+}$and $e^{-}$beams result in a Lorentz boost $\beta \gamma \approx$ 0.55 of the $B \bar{B}$ pairs.

A detail description of the BABAR detector is presented elsewhere [6]. The momenta of the charged particles are measured in a tracking system consisting of a 5-layer double sided silicon vertex tracker (SVT) and a 40-layer drift chamber (DCH). The SVT and DCH operate within a $1.5 \mathrm{~T}$ solenoid field and have a combined solid angle coverage in the center of mass frame of 90.5\%. A detector of internally reflected Cerenkov radiation (DIRC) is used for charged particle identifications of pions, kaons, and protons with likelihood ratios calculated from $d E / d x$ measurements in the SVT and DCH. Photons and long-lived neutral hadrons are detected and their energies are measured in a $\mathrm{CsI}(\mathrm{Tl})$ electromagnetic calorimeter (EMC). For electrons, energy lost due to bremsstrahlung is recovered from deposits in the EMC.

\section{ANALYSIS}

We select charm hadron candidates $X_{c}$ with center of mass frame momentum greater than 2.5 $\mathrm{GeV} / \mathrm{c}$ to suppress combinatoric background. The $e^{+} e^{-}$invariant mass is required to be greater than $200 \mathrm{Mev} / \mathrm{c}^{2}$ in order to reject photon conversion and $\pi^{0}$ decays to $e^{+} e^{-} \gamma$. For the $D_{(s)}^{+} \rightarrow \pi \phi$, $\phi \rightarrow \ell^{+} \ell^{-}$decay mode, we excluded events with $0.95<m\left(e^{+} e^{-}\right)<1.05 \mathrm{GeV} / c^{2}$ and $0.99<$ $m\left(\mu^{+} \mu^{-}\right)<1.05 \mathrm{GeV} / c^{2}$ to reject the decays through the $\phi$ resonance. The QED backgrounds was suppressed by requiring at least five tracks in the event and that hadron candidate be consistent with the electron hypothesis. After the initial event selection, significant combinatorial background contribution, we use three discriminating variables in likelihood ratio: charm hadron candidate, total reconstructed energy in the event, and flight length significance.

To measure the signal events we use extended, unbinned, maximum-likelihood. These signals are converted to the known charm branching fractions by normalization. To reduce the systematic effects we choose normalization modes with kinematics similar to the kinematic of the signal decays. For decays of $D^{+}$and $D_{s}^{+}$mesons, the normalization mode is $\pi^{+} \phi$ where $\phi \rightarrow K^{+} K^{-}$. For the decays of $\Lambda_{c}^{+}$, we choose the decays of $\Lambda_{c}^{+} \rightarrow p K^{-} \pi^{+}$. Figures 2-6 show the fitting results of the invariant mass of $X_{c}^{+} \rightarrow h^{ \pm} \ell^{\mp} \ell^{()^{+}}$decays. The dashed curves show the background components for the dimuon modes in which muon candidates arise from misidentified hadrons. Detail information on the likelihood selection, fitting procedure, systematic uncertainties, and fit results are available here [7]. We calculate the upper limits on the ratio of the branching factions at $90 \%$ confidence level (CL): $\mathscr{B}\left(D_{(s)}^{+} \rightarrow \pi^{ \pm} \ell^{\mp} \ell^{\left({ }^{\prime}\right)+}\right) / \mathscr{B}\left(D_{(s)}^{+} \rightarrow \pi^{+} \phi\right), \mathscr{B}\left(D_{(s)}^{+} \rightarrow K^{ \pm} \ell^{\mp} \ell^{\left({ }^{\prime}\right)^{+}}\right) / \mathscr{B}\left(D_{(s)}^{+} \rightarrow \pi^{+} \phi\right)$, and $\mathscr{B}\left(\Lambda_{c}^{+} \rightarrow p^{(-)} \ell^{\mp} \ell^{\left({ }^{\prime}\right)^{+}}\right) / \mathscr{B}\left(\Lambda_{c}^{+} \rightarrow p K^{-} \pi^{+}\right)$. The most significant signal is seen in the decay of $\Lambda_{c}^{+} \rightarrow p \mu^{+} \mu^{-}$with yield of $11.1 \pm 5.0($ stat $) \pm 2.5$ (syst). It has a statistical significant of $2.6 \sigma$. It is corresponding to $90 \% \mathrm{CL}$ upper limit on the branching fraction of $44 \times 10^{-6}$.

We also recently measured the flavor-changing neutral-current decays $D^{0} \rightarrow e^{+} e^{-}, D^{0} \rightarrow$ $\mu^{+} \mu^{-}$, and $D^{0} \rightarrow e^{ \pm} \mu^{\mp}$ that corresponds to an integrated luminosity of $468 \mathrm{fb}^{-1}$ of data. To normalize the decays of $D^{0} \rightarrow \ell^{+} \ell^{-}$, we use $D^{0} \rightarrow \pi^{+} \pi^{-}$control sample and applying a linear combination of Fisher discriminant [8] of the following five variables: measured $D^{0}$ flight length, $\left|\cos \theta_{\text {hel }}\right|$ angle between the momentum of the positively-charged $D^{0}$ daughter and the boost direction from the lab frame to the $D^{0}$ rest frame (all in the $D^{0}$ rest frame), the missing transverse momentum with respect to the beam axis, the ratio of the $2^{\text {nd }}$ and $0^{\text {th }}$ Fox-Wolfram moments [9], 


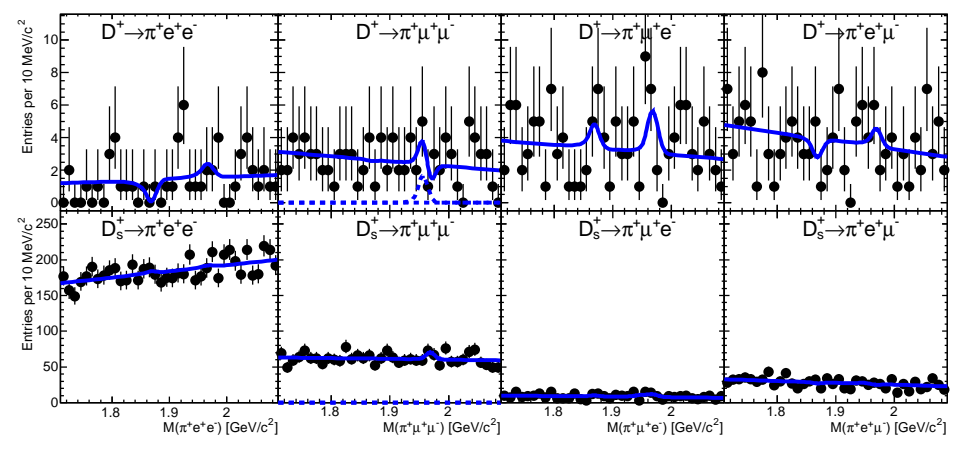

Figure 2: Invariant-mass distributions for $D^{+} \rightarrow \pi^{+} \ell^{+} \ell^{(\prime)}-$ (top) and $D_{s}^{+} \rightarrow \pi^{+} \ell^{+} \ell^{(\prime)}-$ (bottom) candidates. The fit results shown in the solid line.

and the $D^{0}$ momentum in the center of mass frame. To remove the continuum combinatoric background we use the $\left|\cos \theta_{\text {hel }}\right|$ variable. Figure 7 shows distributions of $\left|\cos \theta_{\text {hel }}\right|$ before applying a minimum Fisher discriminant.

The branching fraction of $D^{0} \rightarrow \ell^{+} \ell^{-}$is given by the following expressions:

$$
\mathscr{B}_{\ell \ell}=\left(\frac{N_{\ell \ell}}{N_{\pi \pi}^{\mathrm{fit}}}\right)\left(\frac{\varepsilon_{\pi \pi}}{\varepsilon_{\ell \ell}}\right) \mathscr{B}_{\pi \pi}=S_{\ell \ell} \cdot N_{\ell \ell}
$$

where $S_{\ell \ell}$ is defined by

$$
S_{\ell \ell} \equiv \frac{\mathscr{B}_{\pi \pi}}{N_{\pi \pi}^{\mathrm{fit}}} \frac{\varepsilon_{\pi \pi}}{\varepsilon_{\ell \ell}}
$$

and $N_{\text {obs }}$ is defined by

$$
N_{\text {obs }}=\mathscr{B}_{\ell \ell} / S_{\ell \ell}+N_{B G}
$$

The $N_{\ell \ell}$ and $N_{\pi \pi}^{\mathrm{fit}}$ are the number of $D^{0} \rightarrow \ell^{+} \ell^{-}$and $D^{0} \rightarrow \pi^{+} \pi^{-}$candidates, respectively. The $\mathscr{B}_{\pi \pi}=(1.400 \pm 0.026) \times 10^{-3}$ [10]. We use the likelihood ratio ordering principle of Feldman and Cousins [11] to determine 90\% CL intervals. We find one event of $D^{0} \rightarrow e^{+} e^{-}$with background of $1.0 \pm 0.5$ events and two events of $D^{0} \rightarrow e^{ \pm} \mu^{\mp}$ with background of $1.4 \pm 0.3$ events. These correspond to the $90 \% \mathrm{CL}$ upper limits for the branching fractions $<1.7 \times 10^{-7}$ for $D^{0} \rightarrow e^{+} e^{-}$ and $<3.3 \times 10^{-7}$ for $D^{0} \rightarrow e^{ \pm} \mu^{\mp}$. For the $D^{0} \rightarrow \mu^{+} \mu^{-}$channel, we find eight events with expected background of $3.9 \pm 0.6$. This corresponds to $90 \% \mathrm{CL}$ upper limits on the branching fraction of $[0.6,8.1] \times 10^{-7}$. Detail information on the likelihood selection, fitting procedure, systematic uncertainties, and fit results are available here [12].

\section{CONCLUSIONS}

We have searched for the decay modes $D_{(s)}^{+} \rightarrow \pi^{ \pm} \ell^{\mp} \ell^{(')+}, D_{(s)}^{+} \rightarrow K^{ \pm} \ell^{\mp} \ell^{\left({ }^{\prime}\right)^{+}}$, and $\Lambda_{c}^{+} \rightarrow$ $p^{(-)} \ell^{\mp} \ell^{\left({ }^{\prime}\right)+}$. No signals are observed and we report upper limits on 35 different branching ratios between $0.4 \times 10^{-4}$ and $37 \times 10^{-4}$ at $90 \% \mathrm{CL}$. This corresponds to limits on the branching fractions between $1 \times 10^{-6}$ and $44 \times 10^{-6}$.

We also have searched for the leptonic charm decays $D^{0} \rightarrow e^{+} e^{-}, D^{0} \rightarrow \mu^{+} \mu^{-}$, and $D^{0} \rightarrow$ $e^{ \pm} \mu^{\mp}$. We find no statistically significant excess over the expected background. These results supersede our previous results [13] and are consistent with the results of the Belle experiment [14]. 


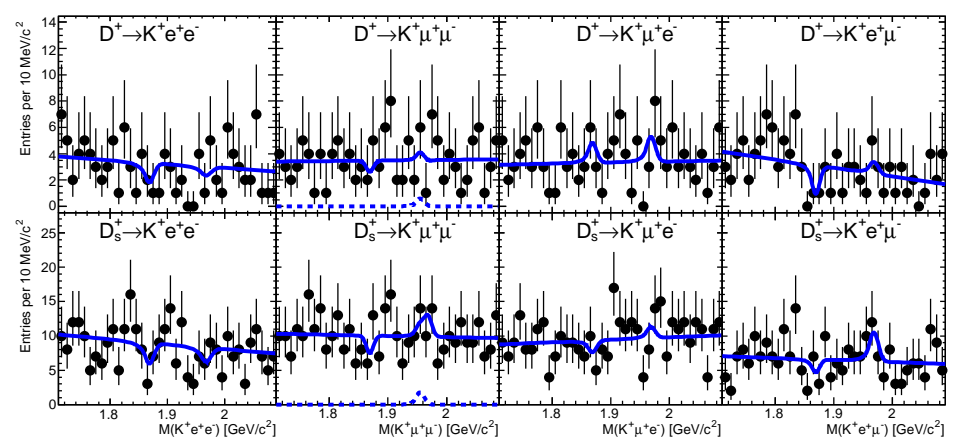

Figure 3: Invariant-mass distributions for $D^{+} \rightarrow K^{+} \ell^{+} \ell^{(\prime)}-$ (top) and $D_{s}^{+} \rightarrow K^{+} \ell^{+} \ell^{(\prime)}-$ (bottom) candidates. The fit results shown in the solid line.

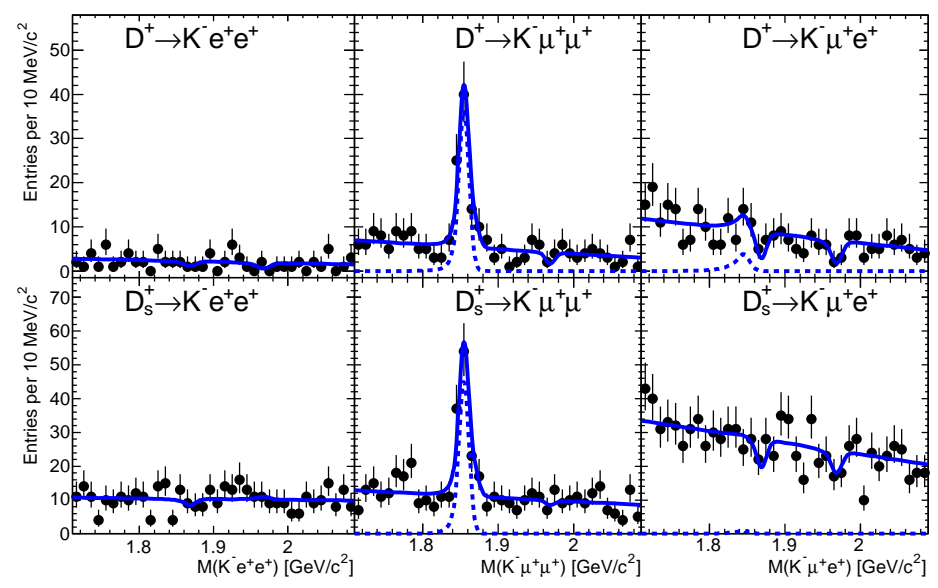

Figure 4: Invariant-mass distributions for $D^{+} \rightarrow K^{-} \ell^{+} \ell^{(\prime)+}$ (top) and $D_{s}^{+} \rightarrow K^{-} \ell^{+} \ell^{(\prime)+}$ (bottom) candidates. The fit results shown in the solid line.

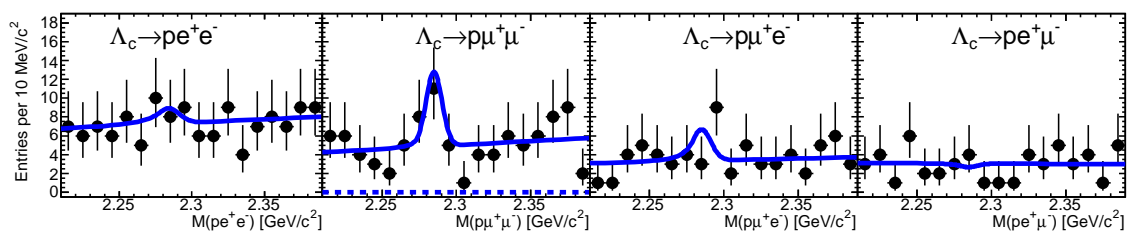

Figure 5: Invariant-mass distributions for $\Lambda_{c}^{+} \rightarrow p \ell^{+} \ell^{(\prime)}-$ candidates. The fit results shown in the solid line.

\section{ACKNOWLEDGMENTS}

The author would like to thank the organizers of ICHEP 2012, the $36^{\text {th }}$ International Conference on High Energy Physics, Melbourne, Australia. The supports from the BABAR Collaboration, the University of South Alabama, and the University of Mississippi are gratefully acknowledged.

\section{References}

[1] S. L. Glashow, J. Iliopoulos, and L. Maiani, Phys. Rev. D 2, 1285, (1970). 


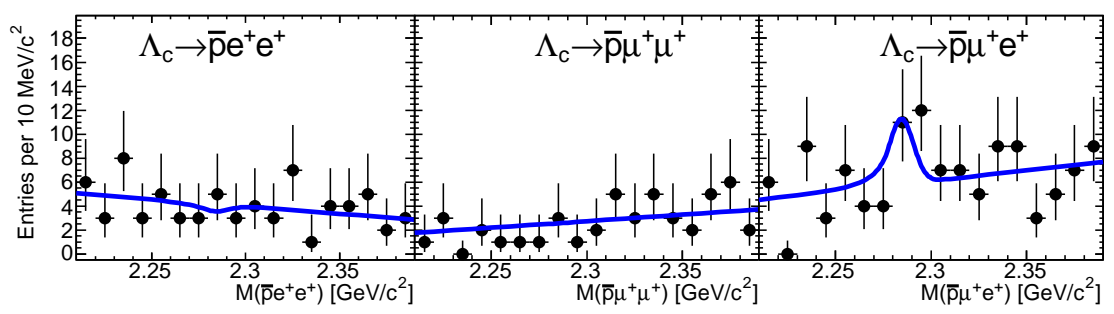

Figure 6: Invariant-mass distributions for $\Lambda_{c}^{+} \rightarrow \bar{p} \ell^{+} \ell^{(\prime)+}$ candidates. The fit results shown in the solid line.
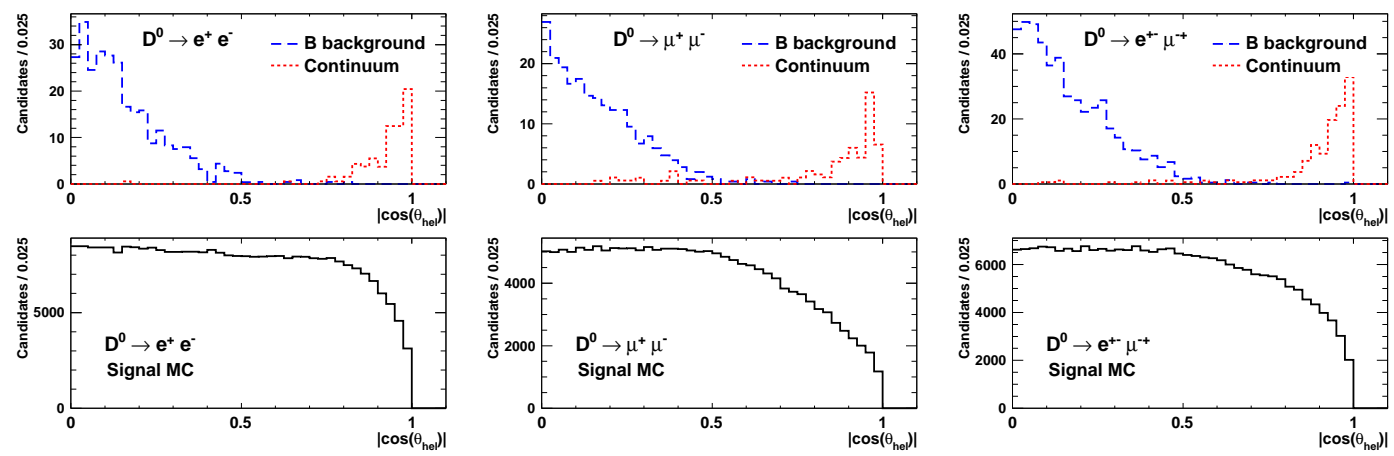

Figure 7: Distributions of $\left|\cos \left(\theta_{\text {hel }}\right)\right|$ for the three signal channels: $D^{0} \rightarrow e^{+} e^{-}$(left), $D^{0} \rightarrow \mu^{+} \mu^{-}$(center), and $D^{0} \rightarrow e^{ \pm} \mu^{\mp}$ (right). The bottom distributions show the signal Monte Carlo samples with an arbitrary normalization.

[2] G. Burdman, E. Golowich, J. Hewett, and S. Pakwasa, Phys. Rev. D 66, 014009, (2002).

[3] S. Fajfer, S. Prelovsek, and P. Singer, Phys. Rev. D 64, 114009, (2001).

[4] E791 Collaboration, E. M. Aitala et al., Phys. Rev. Lett. 86, 3969 (2001).

[5] S. Fajfer, S. Prelovsek, and P. Singer, Phys. Rev. D 58, 094038, (1998).

[6] BABAR Collaboration, B. Aubert et al., Nucl. Instr. Methods Phys. Res., Sect. A 479, 1 (2002).

[7] BABAR Collaboration, J. P. Lees et al., Phys. Rev. D 84, 072006 (2011).

[8] R. A. Fisher, Annals of Eugenics 7, 179 (1936).

[9] G.C. Fox and S. Wolfram, Phys. Rev. Lett. 41, 1581 (1978).

[10] Particle Data Group, K. Nakamura et al., Journal of Phys. G 37, 075021 (2010).

[11] G.J. Feldman and R.D. Cousins, Phys. Rev. D 57, 3873 (1998).

[12] BABAR Collaboration, J. P. Lees et al., Phys. Rev. D 86, 032001 (2012).

[13] BABAR Collaboration, B. Aubert et al., Phys. Rev. Lett. 93, 191801 (2004).

[14] Belle Collaboration, M. Petric et al., Phys. Rev. D 81, 091102(R) (2010). 